\title{
Artigo/Article
}

\section{Talidomida usada por pacientes com eritema nodoso hansênico}

\author{
Thalidomide used by patients with erythema nodosum leprosum
}

\author{
Maria do Socorro da Silva Valente ${ }^{1}$ e José Luiz Fernandes Vieira ${ }^{1}$
}

\begin{abstract}
RESUMO
Introdução: A talidomida é um fármaco utilizado atualmente no tratamento do eritema nodoso hansênico no Brasil. Métodos: Estudo prospectivo para acompanhar a evolução clínica, registrar os eventos adversos e determinar as concentrações plasmáticas de talidomida em dose diária de 100mg/dia, em 20 pacientes com manifestações clínicas de eritema nodoso hansênico, divididos em dois grupos: após ou em curso da poliquimioterapia para hanseníase. Resultados: Não foram observadas diferenças significativas nos grupos no decorrer do estudo, tanto na evolução clínica favorável dos pacientes, de $70 \%$ e $90 \%$, quanto nos eventos adversos registrados que foram tontura e sonolência. Os teores plasmáticos de talidomida em D7 e D14 foram de $0,82 \pm 0,4 \mu \mathrm{g} / \mathrm{mL}$ e $0,79 \pm 0,3 \mu \mathrm{g} / \mathrm{mL}$ no grupo 1 e de $0,82 \pm 0,4$ e 1,55 $\pm 1,0$ no grupo 2, respectivamente. Conclusões: Na amostra estudada, a poliquimioterapia não interferiu na evolução clínica, na incidência dos efeitos adversos e nos níveis plasmáticos de talidomida.
\end{abstract}

Palavras-chaves: Talidomida. Eritema nodoso hansênico. Hanseníase.

\begin{abstract}
Introduction: Thalidomide is a drug currently used in Brazil for treating erythema nodosum leprosum. Methods: This was a prospective study to follow up clinical evolution, record adverse events and determine plasma thalidomide levels from a dose of $100 \mathrm{mg} /$ day, among 20 patients with clinical manifestations of erythema nodosum leprosum, divided into two groups: during or after leprosy multidrug therapy. Results: No significant differences between the groups were seen during the study, either in relation to favorable clinical evolution among the patients (70\% and $90 \%)$, or in relation to the adverse events recorded, which were dizziness and somnolence. The plasma thalidomide levels on D7 and D 14 were $0.82 \pm 0.4 \mu \mathrm{g} / \mathrm{ml}$ and 0.79 $\pm 0.3 \mu \mathrm{g} / \mathrm{ml}$ in group 1 and $0.82 \pm 0.4$ and $1.55 \pm 1.0$ in group 2 , respectively. Conclusions: In this sample, the multidrug therapy had no effect on the clinical evolution, incidence of adverse events and plasma thalidomide levels.
\end{abstract}

Key-words: Thalidomide. Erythema nodosum leprosum. Leprosy.

1. Laboratório de Toxicologia, Faculdade de Farmácia, Universidade Federal do Pará. Belém, PA.

Endereço para correspondência: Dr. José Luiz Fernandes Vieira. Laboratório de Toxicologia/FF/UFPA. Campus Universitário do Guamá. Rua Augusto Corrêa 1, 66075-110 Belém, PA.

Tel: 5591 3201-7733

e-mail: jvieira@ufpa.br

Recebido para publicação em 01/07/09

Aceito em 28/01/2010

\section{INTRODUÇÃO}

O eritema nodoso hansênico (ENH) ocorre em aproximadamente 25 a $30 \%$ dos casos de hanseníase, principalmente na forma multibacilar, de maneira espontânea ou é desencadeado por infecções intercorrentes, anemia, estresse, puberdade, gestação, intervenção cirúrgica e uso de fármacos como antibióticos, progesterona e vitamina $\mathrm{A}^{1-3}$.

Caracteriza-se por lesões eritematosas, dolorosas, de tamanho variável, incluindo pápulas e nódulos, localizadas em diferentes regiões da pele. Alguns casos evoluem com neurite, orquite, epididimite, irite, iridociclite, artrite, linfadenite, dano hepático, edema dos membros inferiores, pré-tibialgia e febre ${ }^{2-4}$.

Dentre os fármacos empregados no tratamento do ENHsedestaca a talidomida (2-phtalimidoglutaramide), liberada para estefim pelo Food andDrugAdministration em 1998, derivada do ácido glutâmico, contendo dois anéis amida e um único centro quiral, portanto uma mistura racêmica, na qual os isômeros $(\mathrm{R})$ e $(\mathrm{S})$ podem apresentar distintas ações farmacológicas ${ }^{5-7}$.

O exato mecanismo de ação da talidomida no ENH permanece incerto; entretanto, ensaios clínicos em processos inflamatórios e oncológicos objetivando esclarecer os múltiplos mecanismos envolvidos na interação deste fármaco com as diferentes linhagens de células do sistema imunológico, retículo-endotelial e nervoso, destacaram sua capacidade de inibir, de maneira seletiva, a produção do fator de necrose tumoral alfa (TNF- $\alpha$ ) em monócitos humanos ${ }^{8,9}$. Neste sentido, pacientes com $\mathrm{ENH}$, apresentam níveis elevados de TNF- $\alpha$ e das interleucinas IL-6, IL-8, IL-10 e proteína C reativa, e após instituição da terapia com talidomida, a regressão dos sinais e sintomas é acompanhada pela diminuição dos teores destes compostos ${ }^{8-10}$.

A dose diária de talidomida recomendada pelo Ministério da Saúde varia de $100 \mathrm{mg}$ a $400 \mathrm{mg}$, de acordo com a gravidade do caso ${ }^{11}$. Nestas doses, não são observados efeitos tóxicos agudos significativos, e os principais efeitos adversos são sonolência, tontura, cefaléia, boca seca e constipação intestinal. 
Ressalta-se que a neuropatia periférica é o principal fator limitante ao uso deste fármaco, é dose dependente, algumas vezes irreversível, e ocorre em cerca de 21 a $50 \%$ dos usuários ${ }^{5,6,12}$. Por outro lado, a teratogenicidade da talidomida requer rigoroso controle do uso por mulheres na idade fértil ${ }^{11,13}$.

Ao se considerar o número reduzido de estudos no Brasil referentes ao uso da talidomida no tratamento do $\mathrm{ENH}$, este trabalho objetivou acompanhar a evolução clínica, registrar os efeitos adversos e quantificar as concentrações plasmáticas de talidomida em pacientes com manifestações clínicas de ENH em curso ou após poliquimioterapia (PQT) da hanseníase, determinando assim, se a PQT interfere nos fatores mencionados.

\section{MÉTODOS}

Foi realizado estudo prospectivo no Ambulatório do Núcleo de Medicina Tropical da Universidade Federal do Pará, referência para o tratamento da hanseníase na cidade de Belém, no período de abril de 2005 a dezembro de 2007. Foram selecionados 20 pacientes, de ambos os sexos, com idade entre 18 e 63 anos, com diagnóstico clínico de $\mathrm{ENH}$, divididos em dois grupos: Grupo 1: 10 pacientes multibacilares (MB), em poliquimioterapia segundo recomendações do Ministério da Saúde para o tratamento de adultos, com dapsona, rifampicina e clofazimina, há pelo menos três meses ${ }^{11}$. Grupo 2: 10 pacientes após uso de poliquimioterapia para hanseníase multibacilar.

A talidomida foi administrada em dose única oral diária de $100 \mathrm{mg}$, à exceção de um paciente do grupo 2, que no décimo primeiro dia após admissão apresentou piora do quadro clínico, sendo necessário aumento da dose para $200 \mathrm{mg} /$ dia. Ao serem inseridos no estudo, todos os pacientes utilizavam prednisona em dose de 1 a $2 \mathrm{mg} / \mathrm{kg} /$ dia, conforme recomendação do Ministério da Saúde ${ }^{11}$, e que não foi considerado critério de exclusão. Não houve relato de uso de outros medicamentos, além daqueles da PQT no grupo 2. Foram seguidas as diretrizes da Lei 10.651, de 16 de abril de 2003, que dispõe sobre o controle do uso da talidomida ${ }^{14}$

Foram excluídos do estudo os pacientes com insuficiência renal ou hepática, com lesões graves que representavam risco à vida ou em tratamento para tuberculose, aids e neoplasias.

\section{Procedimentos}

Foram realizadas três avaliações clínicas e laboratoriais, por duas semanas consecutivas, sendo a primeira para inclusão no estudo (D0), e duas subsequentes, após sete (D7) e quatorze (D14) dias. A primeira, consistiu de anamnese e exame físico, identificando as manifestações clínicas do eritema nodoso hansênico e os efeitos adversos. Já os exames laboratoriais, objetivaram acompanhar a função hepática e a renal.

\section{Exames laboratoriais}

Foram avaliadas as funções renal e hepática, através das determinações de uréia, creatinina, bilirrubinas e transaminases oxalacética e pirúvica. As análises foram realizadas em espectrofotômetro Micronal, com emprego de kits diagnósticos Bioclin ${ }^{\otimes}$

\section{Determinação das concentrações plasmáticas de talidomida}

As amostras de sangue, em volume de $3 \mathrm{~mL}$, foram coletadas em tubos heparinizados, antes da administração da próxima dose de talidomida. Imediatamente centrifugadas a $1.500 \mathrm{~g}$ por 15 minutos.
Precauções foram tomadas referentes à estabilidade da talidomida no plasma após separação, através do tamponamento e armazenamento em condições apropriadas ${ }^{15}$.

A talidomida foi determinada por cromatografia líquida de alta eficiência com detecção no ultravileta (Pro Star - Varian, Walnut, CAUSA). A coluna empregada foi Nucleosil C18 4.6 X250mm (Supelco Inc. Bellefonte PA, USA). A fase móvel foi composta por acetonitrila a $35 \% \mathrm{v} / \mathrm{v}$. Fenacetina $(100 \mu \mathrm{g} / \mathrm{mL})$ foi usada como padrão interno ${ }^{15}$. O procedimento analítico validado demonstrou que o método foi linear em concentrações de 0.044 a 17,5 $\mu \mathrm{g} / \mathrm{mL}$, os coeficientes de variação intra e interdias foram 6,7 e $8,1 \%$, respectivamente. A recuperação média de talidomida foi de $92 \%$.

\section{Análise dos dados}

Para comparação das variáveis avaliadas nos dois grupos foram utilizados o teste exato de Fischer, Kolmogorov-Smirnof e T de Student, com auxílio do programa Biostat 5.0. O nível de significância aceito foi de $5 \%$.

\section{Ética}

Todos os pacientes assinaram o termo de consentimento livre e esclarecido. O projeto obedeceu a resolução 196/96 do Conselho Nacional de Saúde sobre Pesquisa Envolvendo Seres Humanos e foi aprovado pelo Comitê de Ética em Pesquisa do Núcleo de Medicina Tropical da Universidade Federal do Pará, sob o nº 001/2005.

\section{RESULTADOS}

Foi observada prevalência do sexo masculino e da cor parda de $80 \%$ e $70 \%$ no grupo 1 , respectivamente, e de $70 \%$ para as duas variáveis no grupo 2. A faixa etária entre 25 e 34 anos (60\%) e a atividade ocupacional de estudante prevaleceu nos dois grupos de estudo.

Não houve diferença significativa $(p>0,05)$ nos achados clínicos comuns aos dois grupos, prevalecendo febre, nódulos subcutâneos, dores articulares e neurites (Tabela 1). As funções renal e hepática apresentaram valores normais em todos os pacientes nas três avaliações, não representando motivo de exclusão no decorrer do estudo.

Não foi observada diferença significativa na evolução clínica dos pacientes dos dois grupos em D7 e D14 ( $>>0,05)$, a qual foi favorável em torno de 70\% no D7 e de 90\% no D14 (Tabela 2). O paciente que evoluiu de maneira desfavorável apresentou significativa melhora após o aumento da dose de talidomida para $200 \mathrm{mg} /$ dia, assim permanecendo até o final do estudo.

Os efeitos adversos registrados foram relatados pelos pacientes nas duas avaliações clínicas subsequentes à instituição da talidomida. Não foi observada diferença significativa nestes efeitos entre os grupos $(p>0,05)$. Ressalta-se que a constipação intestinal e náuseas foram relatadas apenas no grupo 1 (Tabela 3 ).

Os níveis plasmáticos médios de talidomida em $\mathrm{D} 7$ e $\mathrm{D} 14$ foram de $0,82 \pm 0,4 \mu \mathrm{g} / \mathrm{mL}$ e $0,79 \pm 0,3 \mu \mathrm{g} / \mathrm{mL}$ no grupo 1 e de $0,82 \pm 0,4$ e $1,55 \pm 1,0$ para o grupo 2, respectivamente. Não foram observadas diferenças significativas nos teores plasmáticos médios do fármaco intra grupos e inter grupos nos dias avaliados $(p>0,05)$. O nível plasmático de talidomida do paciente que necessitou aumento da dose para $200 \mathrm{mg} /$ dia não foi considerado para determinação dos teores médios do fármaco do grupo 1 em D14 (Tabela 4). 
TABELA 1 - Manifestações clínicas do eritema nodoso hansênico nos pacientes após (grupo 1) ou no curso (grupo 2) da poliquimioterapia.

\begin{tabular}{lrrrrrrr}
\hline & \multicolumn{2}{c}{ Grupo 1 } & & \multicolumn{2}{c}{ Grupo 2} & Teste de \\
\cline { 2 - 3 } Manifestações clínicas & $\mathrm{n}^{\circ}$ & $\%$ & & $\mathrm{n}^{\circ}$ & $\%$ & Fisher \\
\hline Nódulos subcutâneos & 10 & 100 & & 7 & 70 & 0,2857 \\
Febre & 7 & 70 & & 5 & 50 & 0,5804 \\
Dores articulares & 6 & 60 & & 7 & 70 & 1,0000 \\
Neurite & 5 & 50 & & 7 & 70 & 0,5804 \\
Mialgias & 3 & 30 & & 20 & 1,0000 \\
Cefaléia & 1 & 10 & & - & - & 1,0000 \\
Eritema polimorfo & - & - & & 5 & 50 & 0,0659 \\
\hline no: número de pacientes. & & & & &
\end{tabular}

TABELA 2 - Evolução clínica dos pacientes com eritema nodoso hansênico, após (grupo 1) ou no curso (grupo 2) da poliquimioterapia.

\begin{tabular}{|c|c|c|c|c|c|c|c|c|}
\hline \multirow[b]{4}{*}{ Evolução clínica } & \multicolumn{8}{|c|}{ Avaliações } \\
\hline & \multicolumn{4}{|c|}{$7^{\circ}$ dia } & \multicolumn{4}{|c|}{$14^{\circ}$ dia } \\
\hline & \multicolumn{2}{|c|}{ Grupo 1} & \multicolumn{2}{|c|}{ Grupo 2} & \multicolumn{2}{|c|}{ Grupo 1} & \multicolumn{2}{|c|}{ Grupo 2} \\
\hline & $\mathrm{n}^{\circ}$ & $\%$ & $\mathrm{n}^{\mathrm{o}}$ & $\%$ & $\mathrm{n}^{\mathrm{o}}$ & $\%$ & $\mathrm{n}^{\mathrm{o}}$ & $\%$ \\
\hline Favorável & 7 & 70,0 & 7 & 70,0 & 9 & 90,0 & 8 & 89,0 \\
\hline Estável & 3 & 30,0 & 3 & 30,0 & 1 & 10,0 & - & - \\
\hline Desfavorável & - & - & - & - & - & - & 1 & 11,0 \\
\hline Total & 10 & 100,0 & 10 & 100,0 & 10 & 100,0 & 9 & 100,0 \\
\hline
\end{tabular}

n⿳⺈: número de pacientes, ${ }^{*}$ teste de Kolmogorov-Smirnof: $7^{\circ}$ dia: $\mathrm{p}>0,05 ; 14^{\circ}$ dia: $\mathrm{p}>0,05$.

TABELA 3 - Efeitos adversos associados ao uso de talidomida $100 \mathrm{mg} / \mathrm{dia}$ em pacientes com eritema nodoso hansênico após (grupo 1) ou no curso (grupo 2 ) da poliquimioterapia.

\begin{tabular}{lrrrrrrr}
\hline & \multicolumn{2}{c}{ Grupo 1 } & & \multicolumn{2}{c}{ Grupo 2} & \\
\cline { 2 - 3 } Efeitos adversos & $\mathrm{n}^{\mathrm{o}}$ & $\%$ & & $\mathrm{n}^{\circ}$ & $\%$ & Teste de Fisher \\
\hline Sonolência & 9 & 90,0 & & 10 & 100,0 & 0,2857 \\
Tontura & 2 & 20,0 & 2 & 20,0 & 0,5804 \\
Constipação intestinal & 2 & 20,0 & & - & - & 1,0000 \\
Plenitude gástrica & 2 & 20,0 & & 2 & 20,0 & 0,5804 \\
Náuseas & 1 & 10,0 & & - & - & 1,0000 \\
Cefaléia & 4 & 40,0 & & 2 & 20,0 & 1,0000 \\
\hline
\end{tabular}

no: número de pacientes.

TABELA 4 - Teores médios de talidomida em pacientes com eritema nodoso hansênico após (grupo 1) ou no curso (grupo 2) da poliquimioterapia.

\begin{tabular}{|c|c|c|c|}
\hline \multirow[b]{3}{*}{ Dia } & Grupo 1 & \multicolumn{2}{|c|}{ Grupo 2} \\
\hline & talidomida $\mu \mathrm{g} / \mathrm{mL}$ & tal & ida $\mu \mathrm{g} / \mathrm{mL}$ \\
\hline & $\mathrm{n}^{\mathrm{o}}$ & $\mathrm{n}^{\mathrm{o}}$ & \\
\hline 7 & $0,82 \pm 0,43$ & 10 & $0,82 \pm 0,45$ \\
\hline 14 & $0,79 \pm 0,3$ & 9 & $1,55 \pm 1,06$ \\
\hline
\end{tabular}

no : número de pacientes, ${ }^{*}$ teste $\mathrm{T}$ de student: $\mathrm{p}>0,05$.

\section{DISCUSSÃO}

Os beneficios da talidomida no tratamento do ENH, caracterizados pela regressão das manifestações clínicas e prevenção das incapacidades, sobrepõem a história dramática do seu uso por gestantes, demonstrando a importância dos estudos clínicos e experimentais, visando elucidar a cinética e os vários mecanismos de ação em doenças nas quais o fármaco tem proporcionado resultados excelentes ${ }^{5,12}$.
As características dos pacientes foram semelhantes nos grupos avaliados, demonstrando sua homogeneidade. Houve predomínio do sexo masculino, oito pacientes em cada grupo, o que se associa tanto às precauções e restrições legais ao uso deste fármaco por mulheres na idade fértil, quanto pela prevalência do sexo masculino dentre os portadores de hanseníase ${ }^{1,4,16}$. A faixa etária dos 25 aos 34 anos foi representativa nos dois grupos, corroborando estudos que demonstraram que as reações hansênicas são mais frequentes entre os 20 e 40 anos de idade ${ }^{2,3,9}$.

As manifestações clínicas do ENH ratificam estudos em outros grupos populacionais, entretanto não foi observada redução na freqüência e na severidade das manifestações do ENH nos pacientes do grupo $2^{2,3,9}$.

A evolução clínica do ENH foi similar em ambos os grupos e comparável a outros estudos, que demonstraram melhora clínica, com resposta dramática e rápida, usualmente dentro de um a sete dias após instituição da terapia com talidomida ${ }^{3,5,12}$

Os eventos adversos observados corroboram estudos que indicam que a sonolência, dose dependente, é a principal manifestação no uso da talidomida, que associada à tontura caracterizam a importância da utilização do fármaco em dose única diária antes do adormecer, o que leva a um significativo aumento da aderência ao tratamento ${ }^{3,6,8,12}$.

Os níveis plasmáticos de talidomida de cada grupo nos dias avaliados não apresentaram diferença significante e se assemelham com estudo prévio de dose oral única de $100 \mathrm{mg}$ em pacientes infectados com o vírus da imunodeficiência humana, nos quais o teor médio foi $1.17 \pm 0.21 \mu \mathrm{g} / \mathrm{mL}^{17}$. Também podem ser comparados aqueles realizados em voluntários saudáveis após doses orais únicas de 50 e 200mg do fármaco, no qual as concentrações máximas alcançadas foram $0.62 \pm 0,24$ e $1.76 \pm 0.52 \mu \mathrm{g} / \mathrm{mL}^{18}$.

A ausência de diferença nos níveis séricos de talidomida em $\mathrm{D} 7 \mathrm{e}$ D14 justifica-se pela reduzida meia vida terminal do fármaco, como demonstrado em estudo de doses múltiplas, via oral de talidomida em dose de $200 \mathrm{mg}$ / dia, no qual a meia vida terminal foi de $7.08 \pm 1.87$ e $6.17 \pm 2.56$ horas, respectivamente ${ }^{18}$. Não foi observada diferença significativa nos teores de talidomida entre os grupos, o que pode estar relacionado ao fato do metabolismo enzimático e a excreção renal desempenharem importância secundária na eliminação do fármaco ${ }^{8,17,18}$.

$\mathrm{Na}$ amostra estudada, o uso concomitante da PQT não interferiu na evolução clínica, na incidência dos efeitos adversos e nos níveis plasmáticos de talidomida.

\section{CONFLITO DE INTERESSE}

Os autores declaram não haver nenhum tipo de conflito de interesse no desenvolvimento do estudo.

\section{REFERÊNCIAS}

1. Araújo MG. Hanseníase no Brasil. Rev Soc Bras Med Trop 2003;36:373-382.

2. Chopra NK, Agrawal JS, Pandya PG. Reactions in leprosy. A study of 250 patients in a multidrug therapy project, Baroda District, Gujarat India. Int J Dermatol 1990;29:490-493.

3. Nery JA, Vieira LM, de Matos HJ, Gallo ME, Sarno EN. Reactional states in multibacillary Hansen disease patients during multidrug therapy. Rev Inst Med Trop Sao Paulo 1998;40:363-370. 
4. Foss NT. Hanseníase: aspectos clínicos, imunológicos e terapêuticos. An Bras Dermatol 1999;74:113-119.

5. Parikh DA, Ganapati R, Revankar CR. Thalidomide in leprosy: study of 94 cases. Indian J Lepr 1986;58:560-566.

6. Teo SK, Resztak KE, Scheffler MA, Kook KA, Zeldis JB, Stirling DI, et al. Thalidomide in the treatment of leprosy. Microbes Infect 2002;4:1193-1202.

7. Teo SK, Stirling DI, Zeldis JB. Thalidomide as a novel therapeutic agent: new uses for an old product. Drug Discov Today 2005;10:107-115.

8. Eriksson T, Bjorkman S, Hoglund P. Clinical pharmacology of thalidomide. Eur J Clin Pharmacol 2001;57:365-376.

9. Haslett PAJ, Roche P, Butlin CR, Macdonald M, Shrestha N, Manandhar, R et al. Effective treatment of erythema nodosum leprosum with thalidomide is associated with immune stimulation. J Infect Dis 2005;192:2045-2053.

10. Foss NT, Oliveira EB, Silva CL. Correlation between TNF, increase of plasma $\mathrm{C}$-reative protein level and supression of $\mathrm{T}$ lymphocyte response to concavalin A during erythema nodosum leprosum. Int J Lep 1993;61:218-226.

11. Brasil. Ministério da Saúde, Secretaria de Políticas de Saúde. Guia Para o Controle da Hanseníase. $3^{\text {a }}$ edição, Brasília; 2002.

12. Vilahermosa LG, Fajardo Jr TT, Abalos RM, Balagon MV, Tan EV, Cellona RV et al. A randomized double-blind, double-dummy, controlled dose comparison of thalidomide for treatment of erythema nodosum leprosum. Am J Trop Med Hyg 2005;72:518-526.

13. Lockwood DN. The management of erythema nodosum leprosum: current and future options. Lepr Rev 1996;67:253-259.

14. Brasil. Ministério da Saúde. Lei no 10.651 , de 16 de abril de 2003. Dispõe sobre o controle do uso da talidomida. Diário Oficial da União, Poder Executivo, Brasília, DF, 17 abr; seção 1, 2003. p. 25.

15. Lyon AW, Duran G, Raisys VA. Determination of Thalidomide by High Performance Liquid Chromatography: Methodological Strategy for Clinical Trials. Clin Biochem 1995;28:467-470.

16. Vries JL, Perry BH. Leprosy case detection rates by age, sex and polar type under leprosy control conditions. Am J Epidemiol 1985;12:403-413.

17. Piscitelli SC, Figg WD, Hann B, Kelly G,Thomas S, Walker RE. Single-Dose Pharmacokinetics of Thalidomide in Human Immunodeficiency Virus-Infected Patients. Antimicrob Agents Chemother 1997;41:2797-2799.

18. Teo SK, Colburn WA, Thomas SD. Single-dose oral pharmacokinetics of three formulations of thalidomide in healthy male volunteers. J Clin Pharmacol 1999;39:1162-1168. 